\title{
Chapter 2 \\ Structure of the Pediatric Competence Network of the German GPOH (Society of Pediatric Oncology and Hematology)
}

The pediatric oncologists in Germany are running multicenter treatment optimization studies for all kinds of tumors including CNS tumors. They have instituted reference centers for neuropathology, CSF cytology, radiotherapy, and neuroradiology for these studies in order to harmonize the individual results of histological diagnosis, staging, treatment, and response evaluation of their patients who are diagnosed and treated in many different hospitals all over the country $[6,7]$ rather than in few specialized centers. It has been recognized in several German and European studies that the event-free survival (EFS) of the study patients has been improved by a central review process [8]. The author has been working as the leader of the reference center for neuroradiology for more than 20 years. The aim of this publication is to present on one hand an easy way to approximately predict or sometimes diagnose the histology of many pediatric brain tumors from the aspect on imaging examinations, and on the other hand to guide into the different aspects of trial requirements, frequent diagnostic quality problems, and questions from the oncologists that have to be answered by the radiologist and are influencing the planning of imaging procedures.

Clearly, through the past years if not to say meanwhile decades magnetic resonance imaging (MRI) is dominating the imaging in all kinds of central nervous system (CNS) tumors. But certain features on computed tomography (CT) like the density of the solid parts of tumors, corresponding to the cell density on histological examination, bears indispensible information for the differential diagnosis. As the reference evaluation in the pediatric brain tumor trials is based on structural MRI, we will not cover multimodal imaging methods like diffusion tensor MRI, perfusion techniques, or MRI spectroscopy. We also will not cover positron emission tomography (PET) because this is a nuclear medicine method and beyond the scope of a neuroradiologist. 